\title{
Potential benefits of the application of yeast starters in table olive processing
}

\author{
Francisco N. Arroyo-López ${ }^{*}$, Verónica Romero-Gil ${ }^{1}$, Joaquín Bautista-Gallego ${ }^{1}$, \\ Francisco Rodríguez-Gómez ${ }^{1}$, Rufino Jiménez-Díaz" , Pedro García-García ${ }^{1}$, \\ Amparo Querol ${ }^{2}$ and Antonio Garrido-Fernández ${ }^{1}$
}

\author{
${ }^{1}$ Food Biotechnology Department, Instituto de la Grasa, Consejo Superior de Investigaciones Científicas, Seville, Spain \\ ${ }^{2}$ Food Biotechnology Department, Instituto de Agroquímica y Tecnología de Alimentos, Consejo Superior de Investigaciones Científicas, Paterna, Valencia, Spain
}

\section{Edited by: \\ Aldo Corsetti, University of Teramo, Italy}

\section{Reviewed by:}

Giovanna Suzzi, Università degli Studi di Teramo, Italy

Rosanna Tofalo, University of Teramo, Italy

\section{${ }^{*}$ Correspondence:}

Francisco N. Arroyo-López, Food Biotechnology Department, Instituto de la Grasa, Consejo Superior de Investigaciones Científicas, Avda. Padre García Tejero 4, 41012 Seville, Spain.e-mail:fnarroyo@cica.es
Yeasts play an important role in the food and beverage industry, especially in products such as bread, wine, and beer, among many others. However, their use as a starter in table olive processing has not yet been studied in detail. The candidate yeast strains should be able to dominate fermentation, together with lactic acid bacteria, but should also provide a number of beneficial advantages. Technologically, yeasts should resist low $\mathrm{pH}$ and high salt concentrations, produce desirable aromas, improve lactic acid bacteria growth, and inhibit spoilage microorganisms. Nowadays, they are being considered as probiotic agents because many species are able to resist the passage through the gastrointestinal tract and show favorable effects on the host. In this way, yeasts may improve the health of consumers by means of the degradation of non-assimilated compounds (such as phytate complexes), a decrease in cholesterol levels, the production of vitamins and antioxidants, the inhibition of pathogens, an adhesion to intestinal cell line Caco-2, and the maintenance of epithelial barrier integrity. Many yeast species, usually found in table olive processing (Wickerhamomyces anomalus, Saccharomyces cerevisiae, Pichia membranifaciens, and Kluyveromyces lactis, among others), have exhibited some of these properties. Thus, the selection of the most appropriate strains to be used as starters in this fermented vegetable, alone or in combination with lactic acid bacteria, is a promising research line to develop in the near future.

\section{Keywords: yeast starter, probiotic microorganisms, technological characteristics, table olives}

\section{INTRODUCTION}

Yeasts are unicellular eukaryotic microorganisms with an enormous importance in the food and beverage industry. Sugar fermentation by yeasts is a well-known and old technology where carbohydrates are mainly transformed into water, ethanol, $\mathrm{CO}_{2}$, and other minority compounds. The role played by yeasts in a food ecosystem is double as they can either be beneficial or spoilage microorganisms (Querol and Fleet, 2006). In the specific case of table olives, where a low $\mathrm{pH}$ and high salt concentrations are usually obtained in the final products, they can cause the spoilage of fruits by the production of bad odors and flavors, accumulation of $\mathrm{CO}_{2}$ leading to swollen containers, clouding of brines or softening of fruits, which is especially harmful in olive packaging or storage (for a complete review, see Arroyo-López et al., 2008). However, these microorganisms also possess desirable activities with important technological applications in this fermented vegetable. Several authors have studied, among others, the lipolytic (lipase and esterase), $\beta$-glucosidase, catalase, and killer activities of different yeast species related to table olives or oleic ecosystems for their potential use as starters (Marquina et al., 1992; Psani and Kotzekidou, 2006; Hernández et al., 2007; Bevilacqua et al., 2009; Aponte et al., 2010; Rodríguez-Gómez et al., 2010, 2012; RomoSánchez et al., 2010; Bautista-Gallego et al., 2011). Recently, the probiotic potential of table olive related yeasts has begun to be evaluated (Psani and Kotzekidou, 2006; Silva et al., 2011), which clearly improves the commercial value of the product.

The present mini-review deals with the favorable use of yeasts in table olive processing, focusing on their technological and probiotic applications, especially in those elaborations where their presence is more abundant such as in directly brined green and natural black olives. As olives are not lye treated in these preparations, lactic acid bacteria are partially inhibited due to the presence of phenolic compounds, so the role played by yeasts is more important (Ruiz-Barba et al., 1993; Garrido-Fernández et al., 1997).

\section{USE OF YEAST STARTERS WITH TECHNOLOGICAL APPLICATIONS IN TABLE OLIVE PROCESSING}

The application of yeasts in table olives could technologically improve the process in different ways:

(i) Yeasts produce compounds such as ethanol, glycerol, higher alcohols, esters, and other volatile compounds which may play an important role in flavor generation and texture maintenance during table olive processing (Garrido et al., 1995). In this way, Sánchez etal. (2000) and Montaño et al. (2003) reported the formation of acetic acid, succinic acid, formic acid, ethanol, and acetaldehyde, all of them usually produced by yeasts, during green olive fermentations. The esterase and lipase activities 
exhibited by diverse yeast species could also improve the aromatic profile of fermented olives by increasing their free fatty acid content (Hernández et al., 2007; Rodríguez-Gómez et al., 2010, 2012). In fact, Candida boidinii has been related to a modification of the characteristics of the olive fat during ripe olive processing (Rodríguez-Gómez et al., 2010).

(ii) Biodegradation of polyphenols by yeasts ( $\beta$-glucosidase activity) is another interesting technological aspect. Olives contain high levels of oleuropein, which is responsible for their bitter taste, so they must be treated with a $\mathrm{NaOH}$ solution to make them edible. The presence of yeasts able to biologically degrade phenols could reduce the large quantities of olive wastewater produced during the lye treatment for fruit debittering, leading to a more natural product at the same time.

(iii) Yeasts could also be used as biocontrol agents (Viljoen, 2006). They produce toxic proteins or glycoproteins, also called killer factors, able to inhibit the growth of fungi and other non-desirable yeast species. Wickerhamomyces anomalus (previously called Pichia anomala) and Pichia membranifaciens are species with promising perspectives in this aspect because of their proved inhibitory activity against a considerable number of microorganisms (Santos etal., 2000; Passoth et al., 2011). Therefore, they may reduce the requirements for salt and preservatives necessary to ensure packaging stability and produce healthier products.

(iv) It has been proven that yeasts could improve lactic acid bacteria growth and as well as the production of lactic acid (Tsapatsaris and Kotzekidou, 2004; Segovia Bravo et al., 2007). Yeasts appear to be active microorganisms synthesizing substances such as vitamins, amino acids and purines, or breakdown complex carbohydrates, which is essential for the growth of Lactobacillus species that need a more complex medium for optimal growth (Ruiz-Barba and Jiménez-Díaz, 1995; Viljoen, 2006).

(v) Catalase positive yeast strains are also favorable because they contribute to preserving olives against unsaturated fatty acid oxidation and peroxide formation (Hernández et al., 2007). Fortunately, this is a widespread property in table olive related yeasts, and species such as W. anomalus, Pichia galeiformis, and Kluyveromyces lactis have proven to have this activity (Hernández et al., 2007; Bautista-Gallego et al., 2011).

Therefore, the selection of the most appropriate yeasts to be used as starters must be based on those strains which present the best global activities for the above mentioned characteristics, but they must also be able to predominate during fermentation. They have to present a high resistance to salt and low $\mathrm{pH}$ values. Predictive microbiology is a valuable tool for the discrimination and selection of the most promising strains, determining the influence of environmental variables on yeast growth (Arroyo-López et al., 2010). Another additional problem is the application of an appropriate methodology for the management of such a large amount of data, which is necessary when researchers have to analyze several biochemical activities or growth data (variables) from a considerable number of strains (cases). Multivariate analysis techniques offer a viable approach to solving this setback. Bevilacqua et al. (2009) used a multivariate analysis approach to jointly study growth and qualitative activity data of a number of yeasts isolated from Bella di Cerignola table olives. The use of a principal component analysis was also a valuable tool for the selection and discrimination of several W. anomalus and C. boidinii isolates with the highest global desirable activity levels, clearly differentiated from the rest of assayed yeast species (Rodríguez-Gómez et al., 2012).

\section{USE OF YEAST STARTERS WITH PROBIOTIC CHARACTERISTICS IN TABLE OLIVE PROCESSING}

Probiotics are microorganisms that, after delivery to the gastrointestinal tract, exert beneficial effects to the host $(\mathrm{FAO} / \mathrm{WHO}$, 2001). They should also be safe for the host and metabolically active within the gastrointestinal tract. Probiotics may be consumed either as food components or as non-food preparations.

Table olives are currently being studied as a food carrier whereby probiotic microorganisms could enter the human body. These studies have been mainly focused hitherto exclusively on lactic acid bacteria (Lavermicocca et al., 2005; De Bellis et al., 2010). However, yeasts are interesting microorganisms that may improve human health (Moslehi-Jenabian et al., 2010). Only Psani and Kotzekidou (2006) and Silva et al. (2011) have recently started to assess the probiotic characteristics of table olive related yeasts. The former authors found diverse Torulaspora delbrueckii and Debaryomyces hansenii strains which tolerated high bile salt concentrations and low $\mathrm{pH}$ values. Culture filtrates of these strains inhibited food borne pathogens such as Listeria monocytogenes, Bacillus cereus, and Salmonella typhimurium. The later authors also found diverse P. membranifaciens and Candida oleophila strains with similar properties but with a different spectrum of inhibition (in this case for Escherichia coli, Salmonella enteritidis, and Staphylococcus aureus). Different yeast species, many of them usually found in table olive processing, such as $D$. hansenii, T. delbrueckii, K. lactis, and Saccharomyces cerevisiae, have shown tolerance to passage through the gastrointestinal tract, inhibition of enteropathogens, adhesion to intestinal Caco2 line cell, and immunostimulatory activity (Pennacchia et al., 2008; Kourelis et al., 2010; Moslehi-Jenabian et al., 2010; EtienneMesmin et al., 2011). However, Saccharomyces boulardii is so far the only yeast with clinical effects and the only yeast preparation with proven probiotic efficiency in double-blind clinical studies (Sazawal et al., 2006).

But there are other beneficial effects for human health that justify the application of yeasts in table olive processing. For example, diverse strains of K. lactis, S. cerevisiae, and Issatchenkia orientalis exhibit great ability to reduce cholesterol serum levels (Kourelis etal., 2010). Phytate has a strong chelating capacity and forms insoluble complexes with divalent minerals of nutritional importance such as zinc, calcium, magnesium, and iron. Humans lack the required enzymes in the gastrointestinal tract for the degradation of phytate complexes. Dephosphorylation of phytate is catalyzed by phytases, which are widespread in yeast species such as I. orientalis, W. anomalus, S. cerevisiae, T. delbrueckii, and K. lactis (Olstorpe et al., 2009; Moslehi-Jenabian et al., 2010). Folates (vitamin B9) are essential cofactors in the biosynthesis of nucleotides and therefore 
crucial for cellular replication and growth. Yeasts contain the folate biosynthesis pathway and produce natural folates, but mammals lack the ability to synthesize them and are therefore dependent on their intake from the diet. S. cerevisiae and Candida glabrata are species with high folate synthesis (Moslehi-Jenabian et al., 2010). Diverse yeast strains isolated from table olives and belonging to the P. membranifaciens and Pichia farinosa species have also shown the capacity to produce B-complex vitamins (Ruiz-Barba and Jiménez-Díaz, 1995; Silva et al., 2011). Yeasts can also synthesize a number of bioactive compounds which can serve as natural antioxidants. The screening of yeasts for free-radical-scavenging activity is an active area of research (Gazi et al., 2001). These authors reported that $W$. anomalus produced the highest activity in a laboratory medium. The production of bioactive antioxidants may retard the oxidative degeneration of fatty substances and improve human health.

In any case, an essential requirement for probiotic yeasts is that they must be able to adhere to the olive epidermis, and thus, be ingested by consumers. Figure 1 clearly shows that certain yeast species, in this specific case $C$. boidinii, have the capacity of adhesion and colonization of olive skin, by means of the formation of complex exopolysaccharide matrixes which embed into the microorganisms, and very likely, improve their viability during the passage through the gastrointestinal tract. Personal data show that in many cases these biofilms are formed by mixed populations of yeasts and lactic acid bacteria. However, the molecular basis of this adhesion and interaction between microorganisms is still unclear.

\section{CONCLUSION}

Harnessing and exploiting the technological and probiotic properties of yeasts in table olive processing require fundamental knowledge on their physiology, ecology, biochemistry, and genetics. Inoculated yeast strains should be molecularly monitored throughout fermentation (in both cover brines and olive surface) to confirm their imposition and presence in the final product, especially relevant in the case of probiotic microorganisms. This consciousness will provide the grounds for improving the strategies

\section{REFERENCES}

Aponte, M., Ventorino, V., Blaiotta, G., Volpe, G., Farina, V., Avellone, G., Lanza, C. M., and Moschetti, G. (2010). Study of green Sicilian table olive fermentations through microbiological, chemical and sensory analyses. Food Microbiol. 27, 162-170.

Arroyo-López, F. N., Bautista-Gallego, J., Rodríguez-Gómez, F., and Garrido-Fernández, A. (2010). "Predictive microbiology and table olives," in Current Research, Technology and Education Topics in Applied Microbiology and Microbial Biotechnology, ed. A. MendezVilas (Badajoz: Formatex Research Center), 1452-1461.

Arroyo-López, F. N., Querol, A., Bautista-Gallego, J., and GarridoFernández, A. (2008). Role of yeasts

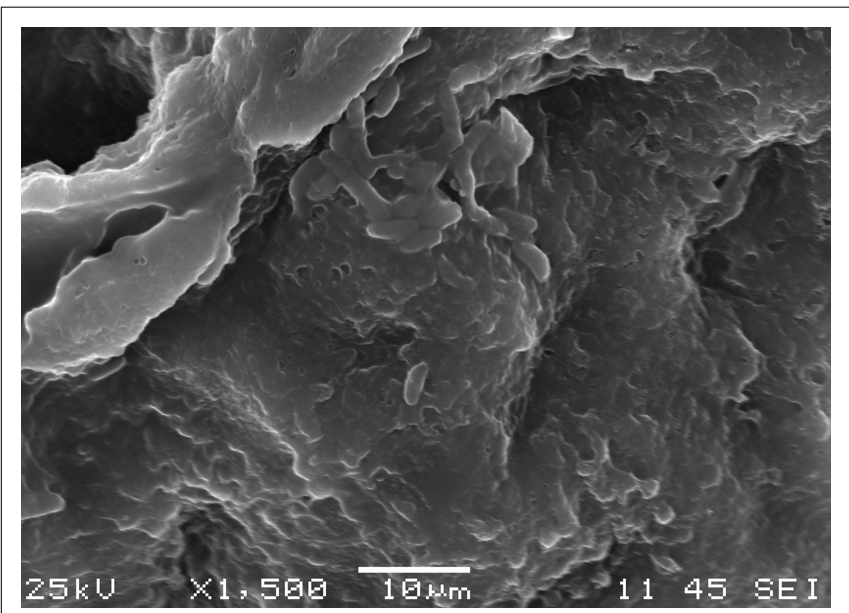

FIGURE 1 | Candida boidinii cells adhering to Gordal olive epidermis. The picture was taken by scanning electronic microscopy and at the bottom, from left to right, shows the voltage used, the scale, the distance in millimeter from microscopy to the sample and the spot size.

performed on this fermented vegetable and increase its competitiveness. Undoubtedly, the full potential of table olive related yeasts has not been fully determined and many challenges are awaiting research, dissemination, and exploitation.

\section{ACKNOWLEDGMENTS}

This research line has received funding from the EU's Seventh Framework Programme [FP7/2007-2013; under grant agreement $\mathrm{n}^{\circ} 243471$ (PROBIOLIVES)]. We also thank the Spanish Government for financial support to projects AGL2009-07436/ALI and AGL2010-15529/ALI, and Junta de Andalucía through financial support to group AGR-125. Thanks to JOLCA and ASEMESA for their invaluable collaboration. Joaquín Bautista-Gallego and Francisco N. Arroyo-López would also like to thank CSIC and Spanish Government for their JAE-PRE fellowship and Ramón y Cajal postdoctoral research contract, respectively.

surface in fermentation sets inoculated with the probiotic strain Lactobacillus paracasei IMPC 2.1 in an industrial plant. Int. J. Food Microbiol. 140, 6-13.

Etienne-Mesmin, L., Livrelli, V., Privat, M., Denis, S., Cardot, J. M., Alric, M., and Blanquet-Diot, S. (2011). Effect of a new probiotic Saccharomyces cerevisiae strain on survival of Escherichia coli O157:H7 in a dynamic gastrointestinal model. Appl. Environ. Microbiol. 77, 1127-1131.

FAO/WHO. (2001). Report on Joint FAO/WHO Expert Consultation on Evaluation of Health and Nutritional Properties of Probiotics in Food Including Powder Milk with Live Lactic Acid Bacteria. Cordoba: FAO/WHO.
Garrido, A., García, P., and Brenes, M. (1995). "Olive fermentation," in Biotechnology: A Multi-Volume Comprehensive Treatise, Vol. 9, eds $\mathrm{H}$. J. Rem and G. Reed (Weinheim: VCH), 593-627.

Garrido-Fernández, A., FernándezDíaz, M. J., and Adams, R. M. (1997). Tables Olives: Production and Processing. London: Chapman \& Hall.

Gazi, M. R., Hoshikuma, A., Kanda, K., Murata, A., and Kato, F. (2001). Detection of free radical scavenging activity in yeast culture. Bull. Fac. Agric. Saga Univ. 86, 67-74. isolated from Bella di Cerignola 2198-2207.

De Bellis, P., Valerio, F., Sisto, A., Lonigro, S. L., and Lavermicocca, P. (2010). Probiotic table olives: microbial populations adhering on olive
Hernández, A., Martin, A., Aranda, E., Pérez-Nevado, F., and Córdoba, M. G. (2007). Identification and characterization of yeast isolated from the elaboration of seasoned green table olives. Food Microbiol. 24, 346-351. 
Kourelis, A., Kotzamanidis, C., Litopoulou-Tzanetaki, E., Scouras, Z. G., Tzanetakis, N., and Yiangou, M. (2010). Preliminary probiotic selection of dairy and human yeast strains. J. Biol. Res. (Thessalon.) 13, 93-104.

Lavermicocca, P., Valerio, F., Lonigro, S. L., De Angelis, M., Morelli, L., Callegari, M. L., Rizzello, C. G., and Visconti, A. (2005). Study of adhesion and survival of Lactobacilli and Bifidobacteria on table olives with the aim of formulating a new probiotic food. Appl. Environ. Microbiol. 71, 4233-4240.

Marquina, D., Peres, C., Caldas, F. V., Marques, F. V., Peinado, J. M., and Spencer, M. (1992). Characterization of the yeast populations in olive brines. Lett. Appl. Microbiol. 14, 279-283.

Montaño, A., Sánchez, A. H., Casado, F. J., de Castro, A., and Rejano, L. (2003). Chemical profile of industrially fermented green olives of different varieties. Food Chem. 82, 297-302.

Moslehi-Jenabian, S., Lindegaard Pedersen, L., and Jespersen, L. (2010). Beneficial effects of probiotic and food borne yeasts on human health. Nutrients 2, 449-473.

Olstorpe, M., Schnüren, J., and Passoth, V. (2009). Screening of yeast strains for phytase activity. FEMS Yeast Res. 9, 478-488.

Passoth, V., Olstorpe, M., and Schnürer, J. (2011). Past, present and future research directions with Pichia anomala. Antonie van Leeuwenhoek 99, 121-125.

Pennacchia, C., Blaiotta, G., Pepe, O., and Villani, F. (2008). Isolation of
Saccharomyces cerevisiae strains from different food matrices and their preliminary selection for a potential use as probiotics. J. Appl. Microbiol. 105 1919-1928.

Psani, M., and Kotzekidou, P. (2006). Technological characteristics of yeast strains and their potential as starter adjuncts in Greek-style black olive fermentation. World J. Microbiol. Biotechnol. 22, 1329-1336.

Querol, A., and Fleet, G. (2006). Yeasts in Food and Beverages. Berlin: SpringerVerlag.

Rodríguez-Gómez, F., Arroyo-López, F. N., López-López, A., BautistaGallego, J., and Garrido-Fernández, A. (2010). Lipolytic activity of the yeast species associated with the fermentation/storage phase of ripe olive processing. Food Microbiol. 27, 604-612.

Rodríguez-Gómez, F., Romero-Gil, V., Bautista-Gallego, J., GarridoFernández, A., and Arroyo-López, F. N. (2012). Multivariate analysis to discriminate yeast strains with technological applications in table olive processing. World J. Microbiol. Biotechnol. 28, 1761-1770.

Romo-Sánchez, S., Alves-Baffi, M., Arévalo-Villena, M., Úbeda-Iranzo, J., and Briones-Pérez, A. (2010). Yeast biodiversity from oleic ecosystems: study of their biotechnological properties. Food Microbiol. 27, 487-492.

Ruiz-Barba, J. L., Brenes, M., JiménezDíaz, R., García-García, P., and Garrido, A. (1993). Inhibition of Lactobacillus plantarum by polyphenols extracted from two different kinds of olive brine. J. Appl. Bacteriol. 74, 15-19.
Ruiz-Barba, J. L., and Jiménez-Díaz, R. (1995). Availability of essential Bgroup vitamins to Lactobacillus plantarum in green olive fermentation brines. Appl. Environ. Microbiol. 61, 1294-1297.

Sánchez, A. H., de Castro, A., Rejano, L., and Montaño, A. (2000). Comparative study on chemical changes in olive juice and brine during green olive fermentation. J. Agric. Food Chem. 48, 5975-5980.

Santos, A., Marquina, D., Leal, J. A., and Peinado, J. M. (2000). ( $1 \rightarrow 6)-\beta$-DGlucan as cell wall receptor for Pichia membranifaciens killer toxin. Appl. Environ. Microbiol. 66, 1809-1813.

Sazawal, S., Hiremath, G., Dhingra U., Malik, P., Deb, S., and Black, R. E. (2006). Efficacy of probiotics in prevention of acute diarrhoea: a meta-analysis of masked, randomised, placebo-controlled trials. Lancet Infect. Dis. 6, 374-382.

Segovia Bravo, K., Arroyo-López, F. N., García-García, P., Duran Quintana, M. C., and Garrido-Fernández, A. (2007). Treatment of green table olive solutions with ozone. Effect on their polyphenol content and on $\mathrm{Lac}$ tobacillus pentosus and Saccharomyces cerevisiae growth. Int. J. Food Microbiol. 114, 60-68.

Silva, T., Reto, M., Sol, M., Peito, A., Peres, C. M., Peres, C., and Xavier-Malcata, F. (2011). Characterization of yeasts from Portuguese brined olives, with a focus on their potentially probiotic behavior. LWT Food Sci. Technol. 44, 1349-1354

Tsapatsaris, S., and Kotzekidou, P. (2004). Application of a central composite design and response surface methodology to the fermentation of olive juice by Lactobacillus plantarum and Debaryomyces hansenii. Int. J. Food Microbiol. 95, 157-168.

Viljoen, B. C. (2006). "Yeast ecological interactions. Yeast-yeast, yeastbacteria, yeast-fungi interactions and yeasts as biocontrol agents," in Yeast in Food and Beverages, eds A. Querol and H. Fleet (Berlin: SpringerVerlag), 83-110.

Conflict of Interest Statement: The author declares that the research was conducted in the absence of any commercial or financial relationships that could be construed as a potential conflict of interest.

Received: 20 March 2012; paper pending published: 06 April 2012; accepted: 10 April 2012; published online: 27 April 2012.

Citation: Arroyo-López FN, Romero-Gil $V$, Bautista-Gallego J, Rodríguez-Gómez $F$, Jiménez-Díaz $R$, García-García $P$, Querol $A$ and Garrido-Fernández A (2012) Potential benefits of the application of yeast starters in table olive processing. Front. Microbio. 3:161. doi: 10.3389/ fmicb.2012.00161

This article was submitted to Frontiers in Food Microbiology, a specialty of Frontiers in Microbiology.

Copyright $\odot 2012$ Arroyo-López,RomeroGil, Bautista-Gallego, Rodríguez-Gómez, Jiménez-Díaz, García-García, Querol and Garrido-Fernández. This is an open-access article distributed under the terms of the Creative Commons Attribution Non Commercial License, which permits non-commercial use, distribution, and reproduction in other forums, provided the original authors and source are credited. 